orbit. The deviation amounts to but $3^{\circ}$, and its plane of rotation has therefore shifted through $177^{\circ}$.

The explanation of the retrograde rotation of Phœbe is now also clear. Phobe, the first-born of Saturn's numerous retinue, came into being while the planet itself still retained its original plane of rotation, that is, while it was still revolving in a retrograde direction. Before Iapetus, Saturn's second satellite, reckoning from without inwards, was created, the mighty tides acting upon the planet in its then diffuse condition had shifted its plane of rotation more than $90^{\circ}$. Two forces then acted on the plane of the orbit of the new satellite, one from the sun tending to bring the orbit into the plane of the orbit of Saturn, the other from Saturn tending to bring the orbit of the satellite into the plane of the equator of its primary. At first both forces tended to produce the same result, namely, to diminish the angle of inclination of the plane of the orbit of the satellite. They are now pulling in opposite directions, as is the case with our own moon, the inclination of the orbit of Iapetus, $19^{\circ}$, being less than that of the equatorial plane of its primary.

The inner satellites of Saturn are more powerfully affected by the equatorial expansion of the planet than by the action of the sun, the planes of their orbits, $27^{\circ}$, coinciding nearly with the plane of the planet's equator.

William H. Pickering.

Harvard Observatory, Cambridge, Mass., U.S.A.

\section{Have Chemical Compounds a Definite Critical Temperature and Pressure of Decomposition?}

So far nobody seems to have considered the question whether to every chemical compound there exists a definite critical temperature and pressure of decomposition. Yet I think the following considerations show that such constants probably do exist. Suppose we place a given compound (say $\mathrm{CaCO}_{3}$ ) in a closed cylinder and subject it to a continually increasing temperature, keeping the pressure constant by means of a weighted piston. Then at a certain definite temperature range the compound will begin to decompose. Suppose, now, we increase the pressure sufficiently; then the decomposition ceases, and the substance can now bear a higher temperature than before without decomposition.

Proceeding in this way, it is, I think, obvious from the finite nature of the mass of the atoms, and from the limited intensity of the forces holding them together in the molecule, that ultimately at some definite finite temperature the external forces tending to drive the atoms apart will become equal to the maximum internal forces that the atoms can exert on each other in the molecule. It therefore follows that above a certain definite temperature, depending upon the nature of the molecule, no pressure, however great, can prevent the substance from completely decomposing. This temperature and pressure, above which a compound is incapable of existing, we will call the critical temperature and pressure of decomposition of the compound. The critical temperature and pressure of decomposition would therefore be completely analogous to the critical temperature of liquefaction of a compoundonly in the latter case we are dealing with the temperature whereat a certain molecular condition of existence disappears, and in the former case with the temperature whereat a certain atomic condition of existence disappears.

Since atoms are a very much more finely divided form of matter than molecules, it is clear that the critical temperature of decomposition of a compound must be a very much sharper and clear-cut constant than its critical temperature of liquefaction. The critical temperature and pressure of even very unstable compounds is usually very high, provided there exist but a few atoms in the molecule. For example, $\mathrm{AuCl}_{3}$, ozone, and the oxides of nitrogen, although very unstable at ordinary temperatures, seem capable of existing at very high temperatures. In general, the greater the number of atoms contained in the molecule the lower the critical temperature of decomposition, as is evident from the general observation that the more complex a compound is the easier it is to decompose. Many of the very complex carbon compounds-for example, the proteids-have, on account of their complexity, critical temperatures of decomposition which lie very close to the normal temperature of the earth's surface.

If, now, by some means we proceed to add on atoms to such a molecule so as to make it more and more complex, we would steadily lower its critical temperature of decomposition, and by adding on a suitable kind and number of atoms we could reduce the critical temperature and pressure of the compound until they coincided with the normal temperatures and pressures which hold upon the earth's surface. Such a compound would be possessed of an extraordinary sensitiveness to external influences on account of the sharpness of the constants called above the critical temperature and pressure of the compound. The slightest increase of temperature or decrease of pressure would serve to throw it into a condition of rapid chemical decomposition, whereas a slight increase of pressure and decrease of temperature would cause it to cease to decompose. Even did we maintain the external temperature and pressure exactly at the critical temperature and pressure of the compound, nevertheless the external impulses which are continuously pervading all space in the neighbourhood of the solar system, beating intermittently upon the sensitive substance, would be sufficient to throw it into a series of rapidly alternating states of decomposition and repose.

I suggest that the temperature range of animal life is probably nothing more or less than the range of the critical temperatures of decomposition of a series of certain very complex carbon compounds which are grouped togethex under the name "protoplasm," the external pressure of the atmosphere coinciding roughly with their critical pressures of decomposition. In fact, I suggest that just as a tuning-fork is set into motion by vibrations of a certain definite frequency and by no others, so living matter is so constructed as to respond continuously to the incessant minute fluctuations in the external conditions which hold upon the earth, the state of response being what is known as life. The temperature of animal life keeps remarkably constant, as it should do on our supposition, a temperature too high exceeding the critical temperature of decomposition of living matter and so destroying its structure, while a temperature too low causes it to cease to decompose, and the living matter becomes inactive.

University of Kiel, April 4.

Geoffrey Martin.

[THE writer of the above will see his "suggestion" discussed in Lockyer's "Inorganic Evolution," book iii.ED. NATURE.]

\section{Experiment on Pressure due to Waves.}

I HAVE seen both in the Physikalische Zeitschrift (January) and in the Physical Reviez (February) an account of an experiment by Prof. R. W. Wood to demonstrate the pressure due to waves, and which he suggests as a lecture demonstration of the effect observed by Lebedeff and by Nichols and Hull. The same experiment is quoted by Prof. Poynting in his address on this subject to the Physical Society of London (Phil. Mag., April). I venture to suggest that the experiment, which consists in setting a small windmill in motion by means of Leyden jar discharges maintained by a transformer, will bear a different explanation. It was shown long ago (I793) by Kinnersley, of Philadelphia, in his "Electrical Thermometer," that a jar discharge produces in air a violent explosive effect, which we should now explain by the repulsion between constituents of the current in opposite phase to one another. The repulsive force may be very great. I think it is this explosive effect that Prof. Wood shows in the experiment, and not the pressure due to reflection of a continuous train of waves. I do not think that the suggestion is new, but it appears to me that the same cause may account for the disruption which occurs when lightning strikes a building, an instance of which is recorded in NATURE of April I3 (p. 565) in the displacement of some of the blocks of the small pyramid.

Sidney Skinner.

South-Western Polytechnic, Chelsea, April 15.

NO. 1852 , VOJ. $7 \mathrm{I}]$ 